\title{
ON THE LINE GRAPH OF A PROJECTIVE PLANE ${ }^{1}$
}

\section{A. J. HOFFMAN}

1. Introduction. If $G$ is a (finite, undirected) graph, its line graph (also called the interchange graph, and the adjoint graph) is the graph $G^{*}$ whose vertices are the edges of $G$, with two vertices of $G^{*}$ adjacent if the corresponding edges of $G$ are adjacent. Let $\pi$ be a projective plane with $n+1$ points on a line, and let $G(\pi)$ be the bipartite graph whose vertices are the $2\left(n^{2}+n+1\right)$ points and lines of $\pi$, with two vertices adjacent if and only if one of the vertices is a point, the other is a line, and the point is on the line. The graph we shall study is $(G(\pi))^{*}$.

For any graph $G$, let

$$
A(G)=A=\left(a_{i j}\right)= \begin{cases}1 & \text { if } i \text { and } j \text { are adjacent vertices } \\ 0 & \text { otherwise }\end{cases}
$$

$A$ is called the adjacency matrix of $G$, and in recent years there have been several investigations to determine to what extent a regular, connected graph is determined by the characteristic roots of its adjacency matrix. In the case where $G$ is a line graph, the following results have been obtained:

(i) If $G$ is the line graph of the complete bipartite graph on $n+n$ vertices, and $H$ is a regular connected graph on $n^{2}$ vertices such that $A(H)$ has the same characteristic roots as $A(G)$, then $H=G$ unless $n=4$, when there is exactly one exception [9].

(ii) If $G$ is the line graph of the complete graph on $n$ vertices, and $H$ is a regular connected graph on $n(n-1) / 2$ vertices, such that $A(H)$ has the same characteristic roots as $A(G)$, then $H=G$, unless $n=8$, when there are exactly three exceptions $[1],[2],[3],[4],[5]$, [8].

In this paper, we shall prove that if $H$ is a regular connected graph on $(n+1)\left(n^{2}+n+1\right)$ vertices such that $A(H)$ has the same characteristic roots as $A\left((G(\pi))^{*}\right)$, then $H=\left(G\left(\pi_{1}\right)\right)^{*}$, where $\pi_{1}$ is some projective plane of the same order as $\pi$. Thus the characteristic roots of $A\left((G(\pi))^{*}\right)$ do determine the class of graphs $(G(\pi))^{*}$, but do not distinguish between projective planes of the same order.

2. The characteristic roots of $A((G(\pi)) *)$. It is useful first to determine the characteristic roots of $A(G(\pi))$.

Received by the editors October 1, 1963.

1 This research was supported in part by the Office of Naval Research under Contract No. Nonr 3775(00), NR 047040. 
LEMMA 1. A regular connected graph $G$ on $2\left(n^{2}+n+1\right)$ vertices has, as the distinct characteristic roots of $A(G)$,

$$
(n+1), \quad-(n+1), \quad \sqrt{ } n, \quad-\sqrt{ } n
$$

if and only if $G=G(\pi)$, where $\pi$ is a projective plane of order $n$.

Proof. By definition, if $G=G(\pi)$,

$$
A(G)=\left(\begin{array}{cc}
0 & B \\
B^{\mathbf{T}} & 0
\end{array}\right),
$$

where $B$ is a point-line incidence matrix of $\pi$. The characteristic roots of (2.2) are the singular values of $B$ and their negatives. But the singular values of $B$ are $n+1$ and $\sqrt{ } n$ [7].

Conversely, assume $A$ has (2.1) as its distinct characteristic roots. If $A=A(G)$, then [6] $G$ is bipartite, so $A$ is of the form (2.2), where $B$ is a $(0,1)$ matrix with row and column sums equal to $n+1$, and $B B^{\mathbf{T}}$ has all but one characteristic root equal to $n$. Hence $B B^{\mathbf{T}}-n I$ is a nonnegative integral symmetric matrix of rank one with every diagonal entry equal to 1 . This implies $B B^{\mathrm{T}}-n I$ has all entries 1 , i.e., $B$ is the incidence matrix of a projective plane $\pi$ of order $n$.

Another derivation of Lemma 1 is given in the thesis of $R$. R. Singleton [10], in which it is proved that a regular connected graph $H$ of valence $n+1$ and girth 6 has $2\left(n^{2}+n+1\right)$ vertices if and only if $H=G(\pi)$.

LEMMA 2. The distinct characteristic roots of $A\left(G(\pi)^{*}\right)$ are

$$
2 n, \quad-2, \quad n-1 \pm \sqrt{ } n \text {. }
$$

Proof. Let $A=A\left((G(\pi))^{*}\right), B$ be the adjacency matrix for $G(\pi)$. Let $K$ be the $2\left(n^{2}+n+1\right)$ by $(n+1)\left(n^{2}+n+1\right)$ matrix whose rows correspond to the points and lines of $\pi$, and whose columns correspond to the edges of $(G(\pi))^{*}$, i.e., each column of $K$ contains two 1 's, corresponding to an incident point and line of $\pi$, the remaining entries in the column being 0 . Clearly,

$$
K K^{\mathrm{T}}=(n+1) I+B, \quad K^{\mathrm{T}} K=2 I+A .
$$

The distinct characteristic roots of $K K^{\mathrm{T}}$ and $K^{\mathrm{T}} K$ are the same except possibly for 0 . But $K^{\mathrm{T}} K$ is singular, since its rank is at most $2\left(n^{2}+n+1\right)$, while its order is $(n+1)\left(n^{2}+n+1\right) ; K K^{\mathrm{T}}$ is singular, since the sum of the rows of $K$ corresponding to points of $\pi$ minus the sum of the rows of $K$ corresponding to lines of $\pi$ is the zero vector. Thus the distinct eigenvalues of $K K^{\mathrm{T}}$ and of $K^{\mathrm{T}} K$ are the same. Invoking (2.1) then proves (2.3). 
3. THEOREM. If $G$ is a regular connected graph with no edges joining $a$ vertex to itself, if $G$ has $(n+1)\left(n^{2}+n+1\right)$ vertices and the adjacency matrix of $G$ has (2.3) as its distinct eigenvalues, then $G=(G(\pi))^{*}$, for some projective plane $\pi$ of order $n$.

In the lemmas that follow, we assume that $G$ satisfies the hypothesis of the theorem, $A=A(G), J$ is the matrix every entry of which is 1 .

LeMma 3. Let

$$
P(x)=\frac{1}{2}\left(x^{3}-(2 n-4) x^{2}+\left(n^{2}-7 n+5\right) x+2\left(n^{2}-3 n+1\right)\right)
$$

then $P(A)=J$.

Proof. It has been shown [6] that the adjacency matrix of a regular connected graph of valence $d$ on $N$ vertices, with distinct eigenvalues $d, \alpha_{1}, \cdots, \alpha_{t}$, satisfies $P(B)=J$, where

$$
P(x)=N \prod_{i}\left(x-\alpha_{i}\right) / \prod_{i}\left(d-\alpha_{i}\right) .
$$

From (2.3), we then calculate (3.1).

LEMMA 4. If two vertices of $G$ are adjacent, then there are exactly $n-1$ vertices of $G$ adjacent to both. If two vertices of $G$ are not adjacent, then there are no vertices or exactly one vertex adjacent to both.

Proof. Let $i$ be any vertex of $G$. Then $i$ has valence $2 n$, so there are $2 n$ vertices $j_{1}, \cdots, j_{2 n}$ such that $a_{i j_{\ell}}=1, t=1, \cdots, 2 n$. We first show that

$$
\sum_{t}\left(A^{2}\right)_{i j_{t}}=2 n(n-1) .
$$

This follows from (3.1); for the left side of (3.2) is $\left(A^{3}\right)_{i i}$, and by (3.1), $\left(A^{3}\right)_{i i}=2(J)_{i i}+(2 n-4)\left(A^{2}\right)_{i i}-\left(n^{2}-7 n+5\right) A_{i i}-2\left(n^{2}-3 n+1\right)$. But $J_{i i}=1,\left(A^{2}\right)_{i i}=2 n, A_{i i}=0$, and (3.2) follows.

Next, consider the matrix

$$
B=A^{2}-2 n I-(n-1) A .
$$

We shall show that every entry of $B$ is 0 or 1 . Certainly every entry is an integer. Let $i$ be any row of $B$. From the fact that $\sum_{j}\left(A^{2}\right)_{i j}$ $=(2 n)^{2}$, we infer that

$$
\sum_{i} b_{i j}=2 n^{2}
$$


We next evaluate $\sum_{j} b_{i j}^{2}=\left(B^{2}\right)_{i i}$. We have from (3.3)

$$
\begin{aligned}
B^{2}= & A^{4}-2(n-1) A^{3}+\left(n^{2}-6 n+1\right) A^{2} \\
& +4 n(n-1) A+4 n^{2} I .
\end{aligned}
$$

Further, $I_{i i}=1, A_{i i}=0, A_{i i}^{2}=2 n, A_{i i}^{3}=2 n(n-1)$ from (3.2). To evaluate $\left(A^{4}\right)_{i i}$, we use (3.1), with $P(A)=J$, and obtain $A P(A)=A J$ $=2 n J$. Since $A P(A)$ is a fourth degree polynomial in $A$, we can evaluate

$$
\left(A^{4}\right)_{i i}=4 n-2 n\left(n^{2}-7 n+5\right)+2 n(n-1)(2 n-4) .
$$

Putting these expressions in (3.5), we obtain

$$
\left(B^{2}\right)_{i i}=\sum_{j} b_{i j}^{2}=2 n^{2}
$$

From (3.5) and (3.6) we infer that each of the integers $b_{i j}$ is 0 or 1. Recalling the definition of $B$ in (3.3), this proves the second sentence of the lemma. To prove the first sentence, note from (3.2) and (3.3) that $\sum_{t} b_{i j_{t}}=0$. Since each $b_{i j}$ is 0 or 1 , each $b_{i j_{t}}=0$. By (3.3), this proves the first sentence of the lemma.

Lemma 5. $G$ contains $2\left(n^{2}+n+1\right)$ cliques $C_{1}, \cdots, C_{2\left(n^{2}+n+1\right)}$ with the following properties:

(3.7) Each $C_{i}$ contains exactly $n+1$ vertices.

(3.8) Each vertex of $G$ is contained in exactly two $C_{i}$.

(3.9) Each pair of adjacent vertices of $G$ is contained in exactly one $C_{i}$.

Proof. The set of cliques $C_{i}$ will consist of all cliques with $n+1$ vertices, which establishes (3.7). To prove (3.9), let $i$ and $j$ be adjacent vertices of $G$. Let $k$ and $l$ each be adjacent to both $i$ and $j$. If $k$ and $l$ were not adjacent, we would have a violation of the second sentence of Lemma 4 . Hence, the $n-1$ vertices adjacent to both $i$ and $j$ (by the first sentence of Lemma 4) are adjacent to each other. These vertices, together with $i$ and $j$, are the unique cliques with $n+1$ vertices containing $i$ and $j$.

Let $T$ be the total number of $n+1$ cliques, and let us count the number of incidences of cliques with pairs of vertices contained in the clique. This is

$$
T\left(\begin{array}{c}
n+1 \\
2
\end{array}\right)=\frac{1}{2} 2 n(n+1)\left(n^{2}+n+1\right),
$$

for the right-hand side is the total number of pairs of adjacent vertices. This equation yields $T=2\left(n^{2}+n+1\right)$. Thus all that remains to 
be proven is (3.8). Since the valence of each vertex $i$ is $2 n$, there must be at least two $n+1$ cliques containing $i$. If these two cliques did not contain all vertices adjacent to $i$, there would have to be some vertex $j \neq i$ in both cliques, violating (3.9).

We are now ready to prove the theorem. Let $\tilde{G}$ be the graph whose vertices are the $n+1$ cliques of $G$. Two vertices of $\tilde{G}$ are adjacent if the corresponding cliques of $G$ have a common vertex. It follows from Lemma 5 that $\tilde{G}$ is a regular connected graph of valence $n+1$, and that $G=\tilde{G}^{*}$. We will be finished if we prove that $\tilde{G}=G(\pi)$. Let $L$ be the vertex-edge incidence matrix of $\tilde{G}$, and let $\tilde{A}$ be the adjacency matrix of $\tilde{G}$. Assume $\tilde{A}$ has distinct characteristic roots $n+1$, $\alpha_{1}, \cdots, \alpha_{t}$. Since

$$
L L^{\mathrm{T}}=(n+1) I+\tilde{A}, \quad L^{\mathrm{T}} L=2 I+A,
$$

and (except possibly for 0 ) the distinct characteristic roots of $L L^{\mathbf{T}}$ and $L^{\mathrm{T}} L$ are the same, it follows by the same reasoning as in Lemma 2 that the distinct characteristic roots (with the possible exception of -2 ) of $A$ are

$$
2 n, \quad n-1+\alpha_{t} .
$$

Comparing (3.10) with (2.3), we see that, if -2 is of the form $n-1+\alpha_{e}$, then $\tilde{A}$ has the same distinct characteristic roots as the adjacency matrix for $G(\pi)$, and (by the "only if" part of Lemma 1) we are finished. Therefore, assume otherwise, so that (comparing (3.10) with (2.1)) we find that the distinct characteristic roots of $\widetilde{A}$ are

$$
n+1, \quad \pm \sqrt{ } n
$$

Since $\tilde{G}$ is regular and connected, we can, as in Lemma 3 , use the theorem of [6] to assert that

$$
2\left(\tilde{A}^{2}-n I\right)=J \text {. }
$$

But since $\tilde{A}$ is a $(0,1)$ matrix, this is absurd.

\section{REFERENCES}

1. L. C. Chang, The uniqueness and nonuniqueness of the triangular association scheme, Sci. Record 3 (1959), 604-613.

2. - Association schemes of partially balanced block designs with parameters $\nu=28, n_{1}=12, n_{2}=15$ and $p_{11}^{2}=4$, Sci. Record 4 (1960), 12-18.

3. W. S. Connor, The uniqueness of the triangular association scheme, Ann. Math. Statist. 29 (1958), 262-266.

4. A. J. Hoffman, On the uniqueness of the triangular association scheme, Ann. Math. Statist. 31 (1960), 492-497. 
5. - On the exceptional case in a characterization of the arcs of a complete graph, IBM J. Res. Develop. 4 (1961), 487-496.

6. - On the Polynomial of a graph, Amer. Math. Monthly 70 (1963), 30-36.

7. H. J. Ryser, Geometries and incidence matrices, Amer. Math. Monthly 62 (1955), No. 7, part II, 25-31.

8. S. S. Shrikhande, On a characterization of the triangular association scheme, Ann. Math. Statist. 30 (1959), 39-47.

9. - The uniqueness of the $L_{2}$ association scheme, Ann. Math. Statist. 30 (1959), 781-798.

10. R. R. Singleton, On minimal graphs of even girth, Thesis, Princeton University, Princeton, N. J., 1962.

International Business Machines Corporation, Research Center, YoRKTOWN HeIGHTS, NEw YORK 